\title{
AMPA Receptor Binding Cleft Mutations That Alter Affinity, Efficacy, and Recovery from Desensitization
}

\author{
Antoine Robert, ${ }^{1}$ Neali Armstrong, ${ }^{2}$ J. Eric Gouaux, ${ }^{2}$ and James R. Howe ${ }^{1}$ \\ ${ }^{1}$ Department of Pharmacology, Yale University School of Medicine, New Haven, Connecticut 06520, and 2Department of Biochemistry and Molecular \\ Biophysics, Columbia University, New York, New York 10032
}

\begin{abstract}
Glutamate binds to AMPA receptors within a deep cleft between two globular protein domains (domains 1 and 2). Once glutamate binds, the cleft closes, and agonist-bound structures of the isolated ligand binding core suggest that closure of the binding cleft is sufficiently complete that it essentially prevents ligand dissociation. There is also considerable evidence supporting the view that cleft closure is the initial conformational change that triggers receptor activation and desensitization, and it has been clearly demonstrated that there is a correlation between the degree of cleft closure and agonist efficacy. It is unknown, however, whether the stability of binding cleft closure also influences receptor-channel properties. The crystallographic structures indicate that closed-cleft conformations are stabilized by the formation of hydrogen bonds that involve amino acid side chains of residues in domains 1 and 2 . We show here that mutations that disrupt one such cross-cleft hydrogen bond (in the AMPA receptor subunit GluR2) decrease both agonist affinity and efficacy. The same mutations also hasten recovery from desensitization. We conclude that the stability of binding cleft closure has a significant impact on AMPA receptor function and is a major determinant of the apparent affinity of agonists. The results suggest that the stability of cleft closure has been tuned so that glutamate dissociates as rapidly as possible yet remains a full agonist.
\end{abstract}

Key words: glutamate; AMPA receptor; desensitization; binding cleft; affinity; mutations

\section{Introduction}

Glutamate is the main excitatory neurotransmitter in the mammalian CNS and has been implicated in a wide variety of brain functions and neuropathologies. The AMPA subfamily of glutamate receptors (GluRs) are ligand-gated ion channels and play a key role in synaptic signaling that occurs on a submillisecond time scale (Dingledine et al., 1999). Recently, structure-function studies have identified important conformational rearrangements in the ligand binding domain that begin to explain how the binding of glutamate to this extracellular domain results in increased ion permeation at the level of the channel pore.

Structures of the GluR2 ligand binding core in conjunction with various ligands confirmed previous suggestions that the glutamate binding pocket is formed from two globular domains that close around agonists in a "venus flytrap" or "hinged clamshell" mechanism (Mano et al., 1996; Dingledine et al., 1999). Competitive antagonists such as DNQX produce minimal domain closure, whereas full agonists such as glutamate, AMPA, and quisqualate cause domain 2 to move $\sim 20^{\circ}$ (Armstrong and Gouaux, 2000; Jin et al., 2002). Partial agonists produce intermediate amounts of binding cleft closure (Armstrong et al., 1998; Jin et al., 2003). The available evidence indicates that the amount of do-

Received Feb. 4, 2004; revised Feb. 18, 2005; accepted Feb. 21, 2005.

This work was supported by the National Institutes of Health (J.R.H., J.E.G.) and the Jane Coffins Memorial Fund for Medical Research (N.A.). We thank Yan Jin for technical assistance, Rebecca Klein for helpful discussions, and Mark Mayer for providing us with the GluR2-wt vector.

Correspondence should be addressed to James R. Howe, Department of Pharmacology, Yale University School of Medicine, 333 Cedar Street, New Haven, CT 06520. E-mail: james.howe@yale.edu.

D01:10.1523/JNEUROSCI.0188-05.2005

Copyright $\odot 2005$ Society for Neuroscience $\quad$ 0270-6474/05/253752-11\$15.00/0 main closure is positively correlated with agonist efficacy and the propensity of ligands to cause receptor desensitization (Armstrong and Gouaux, 2000; Sun et al., 2002; Armstrong et al., 2003; Jin et al., 2003).

AMPA receptors are dimers of dimers (Armstrong and Gouaux, 2000; Ayalon and Stern-Bach, 2001; Mansour et al., 2001; Robert et al., 2001), and several sequence elements and mutations that alter receptor desensitization map to the dimer interface, as does the binding site for cyclothiazide, a compound that greatly slows AMPA receptor desensitization (Armstrong and Gouaux, 2000; Sun et al., 2002). It was proposed that binding cleft closure puts strain on the dimer interface and that desensitization involves rearrangements that relieve this strain (Sun et al., 2002). Although this proposal implies that binding cleft closure gives rise to an inherently unstable transition state, such a state has not been incorporated in previous kinetic models of AMPA receptor gating, and how its stability would impact channel properties has not been considered. For glutamate and other agonists, binding cleft closure is in part stabilized by the formation of hydrogen bonds between residues on opposite sides of the binding cleft. Here we make mutations predicted to disrupt one of these cross-cleft interactions. Our results indicate that decreasing the stability of cleft closure can influence several important properties of AMPA receptors.

\section{Materials and Methods}

Molecular biology. The T686 mutations were incorporated into a GluR2flip construct engineered to delete the $\mathrm{N}$-terminal domain (R2_AATD_m) with the QuikChange protocol (Stratagene, La Jolla, CA). The primers used were as follows: T686A, $5^{\prime}$-CTGTGTTTGTGAG- 
GACCGCCGCAGAAGGAGTAGC-3'; 3'-GCTACTCCTTCTGCGGCGGTCCTCACAAACACAG-5'; and T686S, 5'-CTGTGTTTGTGAGGACCTCCGCAGAAGGAGTAGC-3'; 3'-GCTACTCCTTCTGCGGAGGTCCTCACAAACACAG-5'

Putative mutants were selected by diagnostic restriction digests and verified by sequencing. The mutations were shuttled into full-length GluR2 (flip splice variant with a glutamine at the Q/R editing site) as XhoI/BspEI restriction fragments. The full-length GluR2 construct was kindly provided by Mark Mayer (National Institutes of Health, Bethesda, $\mathrm{MD}$ ) in a cytomegalovirus-driven expression vector modified to also direct expression of enhanced green fluorescent protein (pRK5IEGluRBi).

Electrophysiology. Coverslips of tsA201 cells were transiently transfected with $25 \mu \mathrm{l}$ of a solution consisting of $200 \mu \mathrm{l}$ of Opti-MEM medium (Invitrogen, Gaithersburg, MD), $3 \mu \mathrm{l}$ of Lipofectamine 2000 (Invitrogen), and $1 \mu \mathrm{g}$ of GluR2 plasmid. Patch-clamp recordings were performed $24-72 \mathrm{~h}$ after transfection at room temperature with an EPC 9 amplifier (Heka Elektronik, Lambrecht/Pfalz, Germany) as described previously (Robert et al., 2001). All recordings were from excised outside-out patches, and the holding potential was always set to $-90 \mathrm{mV}$. Series resistance compensation was used routinely and set at $60-80 \%$. The external solution contained the following (in mM): $150 \mathrm{NaCl}, 3 \mathrm{KCl}$, $2 \mathrm{CaCl}_{2}, 1 \mathrm{MgCl}_{2}$, and 5 glucose, buffered with 10 HEPES, $\mathrm{pH}$ adjusted to 7.4 with $\mathrm{NaOH}$. Patch pipettes (open tip resistance of 2-4 M $\Omega$ ) were filled with a solution containing the following (in mM): $120 \mathrm{KF}, 33 \mathrm{KOH}$, $2 \mathrm{MgCl}_{2}, 1 \mathrm{CaCl}_{2}, 0.1$ spermine, and 11 EGTA, pH adjusted to 7.4 with $\mathrm{CsOH}$. Glutamate, quisqualate, and cyclothiazide were added to the external solution. For glutamate-containing solutions, the $\mathrm{NaCl}$ concentration was adjusted so that the total $\mathrm{Na}^{+}$concentration $(200 \mathrm{~mm})$ was the same for all glutamate concentrations. For concentration-response data obtained after slowing desensitization with cyclothiazide, agonists were applied with a perfusion system consisting of a glass pipette containing 12 capillaries connected to different solution reservoirs. Solutions were switched with a series of solenoid valves controlled by the acquisition software (Pulse; Instrutech, Port Washingon, NY) of the patch-clamp amplifier. In experiments in which desensitization was intact, agonists were applied with theta pipettes mounted on a piezoelectric bimorph (part number 62003/5H-144D; Morgan Matroc, Shanghai, China). The tips of the pipettes were broken to $\sim 300 \mu \mathrm{m}$, and the width of the theta glass septum was reduced by etching with hydrofluoric acid. Patches were positioned near the interface of the solutions flowing from adjacent barrels, and the interface was moved by applying voltage across the bimorph with a constant voltage source (HVA-100; ALA Scientific Instruments, Westbury, NY). Voltage pulses were triggered with one of the analog-todigital outputs on the EPC 9 and were analog low-pass filtered (500-700 $\mathrm{Hz} ;-3 \mathrm{~dB}$; four-pole Bessel type) to reduce mechanical oscillations of the piezoelectic device. The rate of solution exchange estimated from open-tip potentials was $100-200 \mu$ s. The bath was constantly superfused with normal external solution flowing at a rate of $1 \mathrm{ml} / \mathrm{min}$.

Glutamate- and quisqualate-evoked currents were analog low-pass filtered at $3 \mathrm{kHz}$ (four-pole Bessel type; $-3 \mathrm{~dB}$ ), sampled at $20-50 \mathrm{kHz}$, and written directly to the hard drive of the computer. The digital records were analyzed using Igor software (WaveMetrics, Lake Oswego, OR), and biexponential exponential functions were fitted to the decays of the currents as described previously (Robert et al., 2001). Concentrationresponse data from individual patches were normalized (see Results), and the mean normalized results were fitted with Hill-type functions to obtain $\mathrm{EC}_{50}$ values and values for the Hill coefficient $\left(n_{\mathrm{H}}\right)$.

Recovery data were obtained from two-pulse protocols. The peak amplitude of the second pulse was expressed as a fraction of the peak amplitude of the paired first pulse. Recovery data were pooled from several patches, and the mean data were fitted with the Hodgkin-Huxley equation: $I_{\mathrm{t}}=\left(I_{\max }{ }^{1 / m}-\left(I_{\max }{ }^{1 / m}-I_{0}{ }^{1 / m}\right) \exp (-t / \tau)\right)^{m}$, where $I_{\mathrm{t}}$ is the peak current at a given interpulse interval, $t, I_{\max }$ is the peak current at long interpulse intervals, $I_{0}$ is the current at zero time (the relative amplitude of the plateau current at the end of the first pulse), $\tau$ is the recovery time constant, and $m$ is an exponent whose value corresponds to the number of kinetically equivalent rate-determining transitions that contribute to the recovery time course.
Kinetic modeling and simulations. Kinetic modeling of GluR2-Q channels was done using Monte Carlo simulations with the software package ChannelLab (Synaptosoft, Decatur, GA). All of the simulations started in zero agonist and included the effect of any conditioning or test pulses. Final simulations were run with 20,000 channels. Simulated recovery time courses were calculated as 1 - (probability of finding the channel in one of the desensitized states), which for the channels studied here agreed within $1 \%$ with the probability of finding the channel in the unoccupied closed state.

The kinetic model used for the simulations is shown in Figure $6 a$. The model is identical to the one shown to account for a wide variety of channel behavior for GluR1 and GluR4 channels (Robert and Howe, 2003). The justification for the model and the methods used to arrive at values for the rate constants have been detailed previously (Robert and Howe, 2003). Although results for GluR1 and GluR4 were collected over a wide concentration range, most of the data reported here were collected at high agonist concentrations. We therefore used rate constants that we estimated previously for GluR1 and GluR4 as starting points for the GluR2-Q simulations and adjusted the values as required to reproduce the experimental results in this paper.

Predicting the effect of the mutations on agonist efficacy. Our Monte Carlo simulations indicated that the shifts in $\mathrm{EC}_{50}$ values and speeding of deactivation that we found for the T686 mutants were most simply accounted for by increases in the rate constant $k_{-1}$ in the model in Figure $6 a$ (see Results). This finding was somewhat surprising because the crystal structures of the isolated binding core indicate that T686 does not directly coordinate agonists. However, these structures also suggest strongly that glutamate does not dissociate at any appreciable rate from the closed-cleft conformation, that T686 makes a cross-cleft hydrogen bond with $\mathrm{E} 402$, and that disruption of this bond would be predicted to destabilize cleft closure. These results led us to hypothesize that the rate at which agonists dissociate, what is seen as $k_{-1}$ in our simulations, is determined by the rate at which the binding cleft opens.

To test this hypothesis, and evaluate whether it would be predicted to alter the relative efficacy of glutamate and quisqualate, we treated the binding and gating of each subunit (in the presence of cyclothiazide) as a three step process in which an agonist (A) first binds to an open-cleft conformation of the subunit (R), the cleft closes, and the subunit can then adopt a conformation $(\mathrm{O})$ that allows current flow. This is formalized in the sequential four-state kinetic scheme:

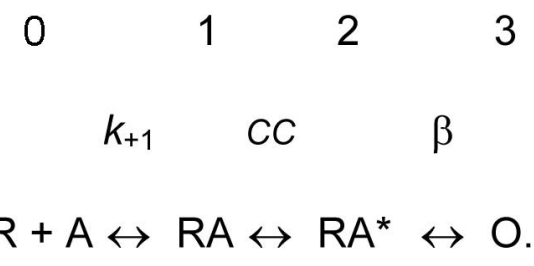

Scheme 1

\section{$\mathrm{R}+\mathrm{A} \leftrightarrow \mathrm{RA} \leftrightarrow \mathrm{RA}^{*} \leftrightarrow \mathrm{O}$.}

$$
k_{-1} \quad \text { co } \quad \alpha
$$

Expanding the model in Figure $6 a$ to include such additional transitions requires the addition of many more states. For example, receptors with four glutamates bound would undergo four sequential cleft closing steps, and receptors with 1,2,3, and 4 clefts closed could open to sequentially larger conductance levels. Including desensitization would require the inclusion of even more states, because desensitization could also occur from any of the four intermediate closed states, and three of those states could undergo two sequential desensitization steps. Given the very large number of free parameters in such complicated models, we elected not to attempt to evaluate our hypothesis with Monte Carlo simulations. Rather we took the simpler approach of assuming each subunit gated independently and then did simple binomial calculations to find the probability that $1,2,3$, or 4 subunits were "open."

For the simple kinetic scheme illustrated above, the probability that a single subunit is "open" is the steady-state probability of finding the subunit in state $\mathrm{O}: P(\mathrm{O})=\left(k_{+1}[\mathrm{~A}] / k_{-1}\right)(C C / C O)(\beta / \alpha) /\left[1+\left(k_{+1}[\mathrm{~A}] /\right.\right.$ 
$\left.k_{-1}\right)\{1+(C C / C O)(1+(\beta / \alpha)\}]$. The hypothesis we wanted to evaluate was that the values of $k_{-1}$ obtained from our Monte Carlo simulations correspond to the rate at which the binding cleft opens. For the calculations of $P(\mathrm{O})$, the values of $C O$ were therefore taken as those estimated for $k_{-1}$ from our Monte Carlo simulations. If $\mathrm{CO}$ is what determines the rate of glutamate dissociation (our hypothesis), then $k_{-1}$ (the rate constant for dissociation from the open-cleft conformation) must be much larger. For $P(\mathrm{O})$ calculations, $k_{-1}$ was set to $100,000 \mathrm{~s}^{-1}$. Values for $k_{+1}$ were set to $1 \times 10^{7} \mathrm{M}^{-1} \mathrm{~S}^{-1}$, assuming that agonist binding to the opencleft conformation is primarily diffusion limited. Although the results of our Monte Carlo simulations were primarily determined by the ratio of $\beta$ and $\alpha$ rather than their absolute values, the value of $\alpha$ that we estimated from simulations $\left(4000 \mathrm{~s}^{-1}\right)$ is consistent with previous measurements of apparent open times for GluR2-Q homomers and was therefore used for calculations of $P(\mathrm{O})$ (Swanson et al., 1997a; Jin et al., 2003) (admittedly not corrected for missed events). To our knowledge, there are no measurements that provide a direct estimate of $C C$, and estimates of $\beta$ depend significantly on the temporal resolution of the data set and vary significantly (Jonas et al., 1993; Raman and Trussell, 1995; Partin et al., 1996; Dzubay and Jahr, 1999; Banke et al., 2000; Koike et al., 2000; Robert et al., 2001; Sekiguchi et al., 2002; Robert and Howe, 2003). We therefore calculated $P(\mathrm{O})$ for multiple values of $C C$ and a range of $\beta$ values.

Assuming that individual subunits gate independently, the probability that $j$ subunits were in state 0 in our simple kinetic scheme is given by the following binomial equation: $P(j)=[N ! /(j !(N-j) !)]\left(P(O)^{j}(1-\right.$ $P(\mathrm{O})^{N-j}$ ), where $N=4$ and $j=1$ to 4 . The $P(j)$ values $(j=1$ to 4 ) were then scaled by the values of the four open levels seen in single-channel records for wild-type GluR2 (GluR2-wt) receptors [9, 16, 20, and $27 \mathrm{pS}$ (A. Robert and J. R. Howe, unpublished observations)], and the scaled values were summed to estimate the relative population responses expected for quisqualate and glutamate at concentrations of 10 and $50 \mathrm{~mm}$, respectively.

\section{Results \\ An interdomain hydrogen bond stabilizes the binding domain in the closed-cleft conformation}

Previous crystal structures of the GluR2 ligand-binding core (S1S2) revealed how glutamate binding leads to closure of the binding domain (Fig. 1) (Armstrong et al., 1998; Armstrong and Gouaux, 2000). The closed-cleft conformation is stabilized by interactions between glutamate and residues from domain 1 and domain 2, as well as by interdomain interactions that occur only in the agonist-bound state. One such interdomain interaction is a hydrogen bond between E402 and T686. Closure of the binding cleft brings T686 in domain 2 within $2.7 \AA$ of E402 from domain 1 ; in the agonist-free or apo state, these side chains are separated by $>6.0 \AA$. Neither E402 nor T686 directly interacts with glutamate, but mutation of E402 increases agonist $\mathrm{EC}_{50}$ values and reduces agonist affinity in binding assays (Uchino et al., 1992; Mano et al., 1996; Lampinen et al., 1998; Abele et al., 2000). Whether these changes reflect true reductions in agonist affinity is not known, however, and how the changes relate to disruption of the E402-T686 interaction has not been considered. To test further the effects of disrupting this interaction on AMPA receptor function, we made mutations at T686.

\section{Mutations at T686 reduce the apparent affinity of glutamate}

Both E402 and T686 project toward the central axis of the binding cleft, lying on opposite sides of its mouth (Fig. 1). Although these amino acids do not coordinate glutamate, the introduction of serine or alanine at position 686 caused a significant rightward shift in the $\mathrm{EC}_{50}$ value for glutamate. Figure $2 a$ shows representative concentration-response data obtained in the presence of cyclothiazide in individual outside-out patches pulled from cells transfected with GluR2-wt or the T686S mutant. Pooled data for GluR2-wt and the T686S and T686A mutants are plotted in Fig-
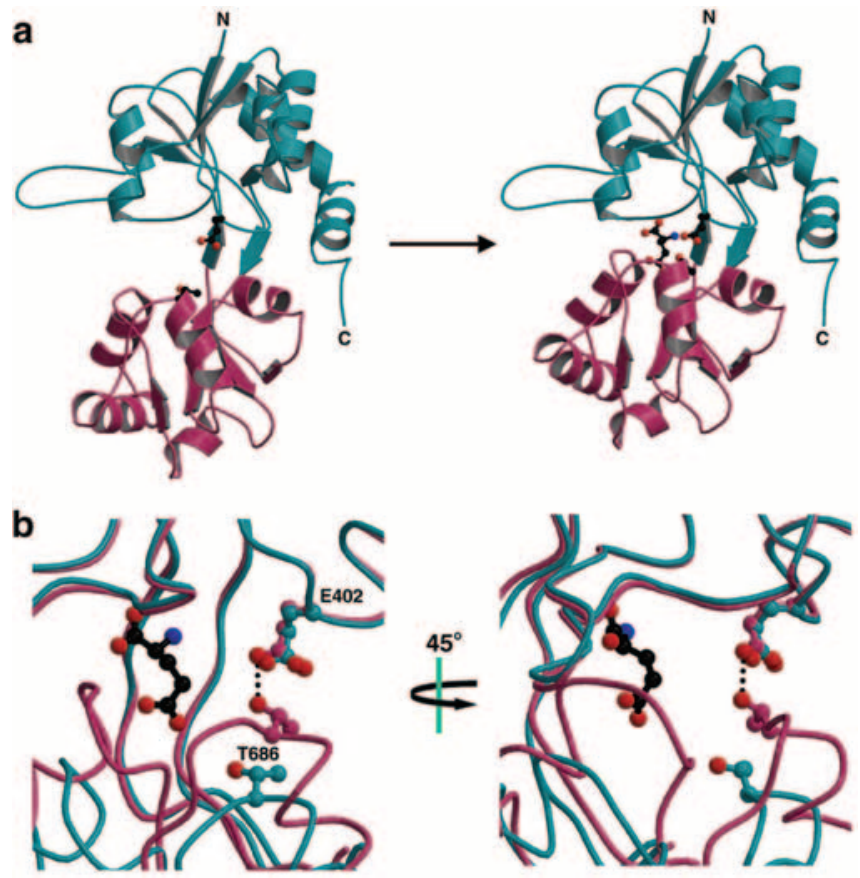

Figure 1. E402 and T686 form an interdomain hydrogen bond only in the glutamate-bound state. $\boldsymbol{a}$, Ribbon diagrams of apo (left) and glutamate-bound (right) GluR2 S1S2 depict the conformational change that occurs during agonist binding. Glutamate binding induces an $\sim 20^{\circ}$ rotation of domain 2 (magenta) toward domain 1 (cyan), bringing T686 within hydrogen bonding distance of E402. E402, T686, and glutamate are drawn in ball-and-stick. C, C terminus; $\mathrm{N}, \mathrm{N}$ terminus. $\boldsymbol{b}$, Superposition of the apo (cyan) and glutamate-bound (magenta) crystal structures illustrates the $>3.0 \AA$ movement of T686 toward E402 that occurs during glutamate binding. In the apo structure, the T686 hydroxyl group is $>6.0 \AA$ from the $\gamma$-carboxyl of E402, whereas in the glutamate structure, these side chains are clearly within hydrogen bonding distance $(\sim 2.8 \AA)$. Notice, also, that neither residue makes any direct or water-mediated contacts with glutamate.

ure $2 b$ (all constructs were "flip" splice variants and contained a glutamine at the $\mathrm{Q} / \mathrm{R}$ editing site). For each recording, the results were normalized to the size of the current evoked by $50 \mathrm{~mm}$ glutamate. The Hill-type fits to the results gave $\mathrm{EC}_{50}$ values of $0.28,3.2$, and $6.4 \mathrm{~mm}$ for GluR2-wt and the serine and alanine mutants, respectively. Consistent with the rightward shifts in $\mathrm{EC}_{50}$ values, the T686 mutations also increased the rate at which the currents decayed at the end of the glutamate applications. This effect is illustrated in Figure $2 c$. Mutations at the residue equivalent to T686 in the kainate receptor subunits GluR5 and GluR6 also alter agonist affinities and deactivation rates (Swanson et al., 1997b, 1998).

\section{The effect of T686 mutations on} glutamate-evoked desensitization

We next compared the responses of the channels to glutamate with desensitization intact. Figure $2 d$ shows examples of the results of this comparison. In agreement with previous studies, single-exponential fits for GluR2-wt channels gave desensitization time constants of $\sim 5 \mathrm{~ms}$ (Koike et al., 2000; Sun et al., 2002). As noted previously for GluR1 channels (Robert et al., 2001), biexponential fits were significantly better, and two exponentials were fitted to all the desensitization decays. These fits gave mean time constants for GluR2-wt of 4.2 and $11.3 \mathrm{~ms}$. Values obtained for the T686S mutant were marginally slower, whereas desensitization decays were approximately twofold slower for the alanine mutant. The T686 mutations also reduced the peak-to-plateau current ratio relative to wild-type channels. The increase in the 
a GluR2-wt

glutamate from $20 \mu \mathrm{M}$ to $50 \mathrm{mM}$

$10 \mathrm{~s}$

c

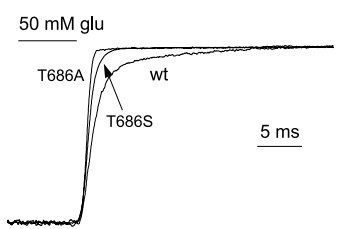

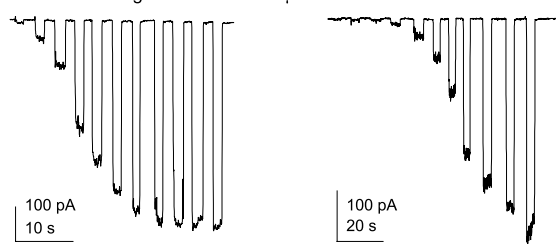

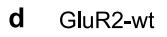

$50 \mathrm{mM} \mathrm{glu}$

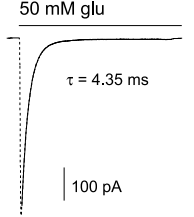

b
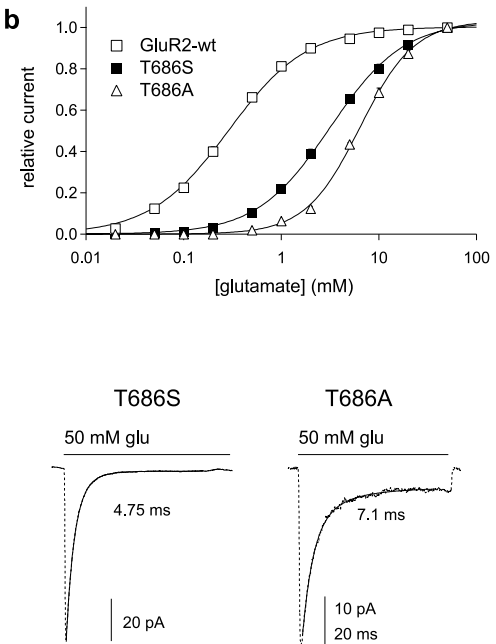

T686A

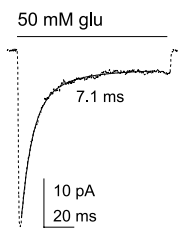

Figure 2. Mutations at T686 reduce apparent affinity but have little effect on the rate of desensitization. $\boldsymbol{a}$, Inward currents evoked by the application of glutamate $(20 \mu \mathrm{m}$ to $50 \mathrm{~mm})$ in the presence of $100 \mu \mathrm{m}$ cyclothiazide in outside-out patches from cells transfected with the cDNAs encoding GluR2-wt and the T686S mutant. $\boldsymbol{b}$, Concentration-response data for GluR2-wt and the T686 mutants. The results are mean data pooled from four to six patches. Current amplitudes were normalized to the size of the current obtained with $50 \mathrm{~mm}$ glutamate in the same patch. In this and subsequent figures, error bars indicate SEM, which was often smaller than one-half the symbol size. Each data set was fitted with a Hill-type equation (smooth curve). The maximum of each curve was not constrained and was slightly $>1.0$ for each of the $T 686$ mutants, indicating that $50 \mathrm{~mm}$ is not quite saturating for these receptors. The $\mathrm{EC}_{50}$ and $n_{\mathrm{H}}$ values obtained from the fits are given in Table 1. c, Decays of the currents through GluR2-wt, T686S, and T686A channels at the end of an application of $50 \mathrm{~mm}$ glutamate ( $\mathrm{glu}$; bar). Each record is the average of 20 consecutive trials, and the mean currents were scaled to have the same steady-state amplitude. The currents were recorded in the continuous presence of $100 \mu \mathrm{m}$ cyclothiazide. $\boldsymbol{d}$, Examples of currents evoked by $100 \mathrm{~ms}$ applications of $50 \mathrm{~mm}$ glutamate (bars) with desensitization intact in outside-out patches from cells expressing GluR2-wt, T686S, and T686A homomeric channels. The decays of the currents were fitted with biexponential functions. The fits are superimposed on the current decays and gave the indicated time constants for the predominant fast component of decay. Each record is the average of 20 consecutive trials, and the mean peak currents were scaled to have the same amplitude. and mutant channels that decayed at similar rates (Fig. $3 c$, Table 1), although the effect of the mutations to decrease the peak-to-plateau current ratio was similar for the two agonists (Fig. $3 d$, Table 1).

We also obtained concentration-response data for peak quisqualate-evoked currents with desensitization intact (Fig. 4). The $\mathrm{EC}_{50}$ and $n_{\mathrm{H}}$ values for GluR2-wt and the T6866 mutants are given in Table 1. Because of the low affinity of the T686 mutants for glutamate, we did not determine $\mathrm{EC}_{50}$ values for peak currents, but the $\mathrm{EC}_{50}$ for GluR2-wt channels was $2.2 \mathrm{~mm}$. For quisqualate, peak currents at concentrations below $100 \mu \mathrm{M}$ are underestimated because activation is slow and some channels desensitize before others activate. It is therefore likely that the true $\mathrm{EC}_{50}$ value for quisqualate and GluR2-wt channels is smaller than that measured $(0.27 \mathrm{~mm})$. With this caveat, the shifts in the $\mathrm{EC}_{50}$ values produced by each mutation are similar in the absence and presence of cyclothiazide.

The quisqualate results argue that the effects of the T686 mutations on gating are minimal, and they suggest that the steeper concentration-response curve and slower desensitization seen with glutamate for T686 channels arise from the very low apparent affinity with which glutamate binds to this mutant. relative amount of steady-state current is evident from inspection of the records in Figure $2 d$, in which the currents have been scaled so that the amplitudes of the peak currents are the same. The desensitization time constants and plateau-to-peak current ratios for the three types of homomeric channel are summarized in Table 1.

\section{The effect of T686 mutations on quisqualate-evoked currents}

The slower desensitization and steeper concentration-response curve seen with the T686A mutant (Fig. 2, Table 1) might reflect qualitative changes in agonist-receptor interactions. For example, disrupting the interaction between T686 and E402 might alter the extent of cleft closure or the nature of glutamate binding. Alternatively, the differences may be related to the very low affinity of the T686A channels for glutamate. To distinguish between these two alternatives, we tested the effect of the mutations on quisqualate-evoked currents. Quisqualate is substantially more potent than glutamate, but the two agonists induce a similar amount of binding cleft closure and interact similarly with residues in the binding cleft (Jin et al., 2002).

Representative and mean concentration-response data for quisqualate (in the presence of $100 \mu \mathrm{M}$ cyclothiazide) are shown in Figure 3, $a$ and $b$. The quisqualate $\mathrm{EC}_{50}$ value for GluR2-wt channels $(17 \mu \mathrm{M})$ is 16-fold smaller than the corresponding value for glutamate. As with glutamate, the T686S and T686A mutations produce rightward shifts in the quisqualate concentrationresponse curve $\left(\mathrm{EC}_{50}\right.$ values of 98 and $515 \mu \mathrm{M}$, respectively), but, in contrast to the results with glutamate, the three curves for quisqualate have similar slopes (Table 1). In addition, sustained applications of quisqualate produced peak currents for wild-type

\section{The T686 mutations speed recovery from desensitization}

The observed increases in the plateau currents suggest that the serine and alanine substitutions progressively destabilize desensitized states and increase the rate of resensitization. To test the effect of the mutations on the rate of resensitization directly, we used conventional two-pulse protocols to compare the rate at which GluR2-wt and the T686 mutants recover from desensitization.

Results with glutamate from individual patches are shown in Figure 5, $a$ and $b$, for GluR2-wt and the T686S mutant, and representative results with quisqualate for GluR2-wt and the T686A mutant are shown in Figure 5, $d$ and $e$ (note the different time scales). Substitution of serine and alanine at T686 progressively hastened recovery from desensitization produced by both glutamate and quisqualate. Mean data from several patches are shown in Figure $5 c$ (glutamate) and Figure $3 f$ (quisqualate), in which, because the recoveries differ substantially, the interpulse intervals are plotted on a logarithmic scale. The results were fitted with Hodgkin-Huxley-type equations to obtain time constants for recovery and $m$ values that give an indication of the extent to which the time course deviated from simple exponential recovery (Bowie and Lange, 2002; Robert and Howe, 2003). The current at zero time was taken as the amplitude of the steady-state glutamate- or quisqualate-evoked current at the end of the first application (expressed as a fraction of the corresponding peak current).

Consistent with previous work (Patneau, 1991), recovery was substantially slower for quisqualate than for glutamate (approximately sixfold in our hands). For quisqualate, the mutations did not alter the shape of the recovery time course, and the relative decreases in the time constant of recovery agreed closely with the 
Table 1. Properties of GluR2-wt and T686 mutants

\begin{tabular}{|c|c|c|c|c|c|c|}
\hline & \multicolumn{3}{|l|}{ Glutamate } & \multicolumn{3}{|l|}{ Quisqualate } \\
\hline & GluR2-wt & T686S & $\mathrm{T} 686 \mathrm{~A}$ & GluR2-wt & T686S & $\mathrm{T} 686 \mathrm{~A}$ \\
\hline $\mathrm{EC}_{50}(\mathrm{~mm})^{a}$ & 0.28 & 3.2 & 6.4 & 0.016 & 0.10 & 0.54 \\
\hline$n_{\mathrm{H}}{ }^{a}$ & 1.05 & 1.15 & 1.55 & 1.34 & 1.40 & 1.22 \\
\hline peak $\mathrm{EC}_{50}(\mathrm{~mm})^{b}$ & 2.2 & n.d. & n.d. & 0.27 & 0.65 & 2.97 \\
\hline$n_{\mathrm{H}}{ }^{b}$ & 1.18 & n.d. & n.d. & 1.08 & 1.18 & 1.24 \\
\hline$\tau_{\text {des } 1}(\mathrm{~ms})^{c}$ & $4.2 \pm 0.1$ & $4.6 \pm 0.3$ & $7.4 \pm 0.7$ & $3.3 \pm 0.2$ & $3.5 \pm 0.2$ & $4.2 \pm 0.3$ \\
\hline$\tau_{\text {des } 2}(\mathrm{~ms})^{c}$ & $11.3 \pm 0.4$ & $14.2 \pm 1.2$ & $18.5 \pm 2.2$ & $8.5 \pm 0.6$ & $10.2 \pm 1.5$ & $12.5 \pm 0.6$ \\
\hline$\tau_{\text {recov }}(\mathrm{ms})^{d}$ & 18.1 & 10.7 & 6.8 & 109 & 21 & 9.7 \\
\hline$m^{d}$ & 2.00 & 1.52 & 0.86 & 2.05 & 2.00 & 1.90 \\
\hline$I_{\text {plat }}(\% \text { of peak })^{e}$ & $0.8 \pm 0.1$ & $1.5 \pm 0.3$ & $14.5 \pm 2.1$ & $0.25 \pm 0.10$ & $1.3 \pm 0.2$ & $2.1 \pm 0.35$ \\
\hline
\end{tabular}

n.d., Not determined.

${ }^{a}$ Values from Hill-type fits to the mean data shown in Figures $2 b$ and $3 b$.

${ }^{b}$ Values from Hill-type fits to concentration-response data for peak currents.

'Mean \pm SEM values from biexponential fits to the current decays during $100 \mathrm{msec}$ applications of $50 \mathrm{~mm}$ glutamate $(n=5-7$ patches). The slower component was $5-20 \%$ of the total decay.

${ }^{d}$ Values from Hodgkin-Huxley-like fits to the mean data shown in Figure 3, $c$ and $f$.

${ }^{e}$ Mean \pm SEM values measured with $50 \mathrm{~mm}$ glutamate ( $n=5-7$ patches).

Table 2. Rate constants for GluR2-wt and the T686 mutants

\begin{tabular}{|c|c|c|c|c|c|c|}
\hline & \multicolumn{3}{|l|}{ Glutamate } & \multicolumn{3}{|c|}{ Quisqualate } \\
\hline & GluR2-wt & T686S & T686A & GluR2-wt & T686S & T686A \\
\hline$\alpha^{a}$ & 4000 & & & & & \\
\hline$\beta^{a, b, c}$ & 5000 & & & & & \\
\hline$k_{+1}^{d}$ & $1 \times 10^{7}$ & & & & & \\
\hline$k_{-1}^{e}$ & $2 \times 10^{4}$ & n.d. & n.d. & 2000 & 4000 & $3 \times 10^{4}$ \\
\hline$\delta_{1}^{b, g}$ & 350 & & & 450 & & \\
\hline$\delta_{2}^{f, g}$ & 1000 & & & & & \\
\hline$\gamma_{1}^{f, g}$ & 60 & 110 & 200 & 10 & 40 & 100 \\
\hline$\gamma_{2}^{f, g}$ & 130 & 250 & 800 & 22 & 90 & 210 \\
\hline$k_{-1}^{* h}$ & 3000 & $3 \times 10^{4}$ & $6 \times 10^{4}$ & 200 & 1000 & 5000 \\
\hline
\end{tabular}

All rate constants have units of $\mathrm{s}^{-1}$, except $k_{+1}\left(\mathrm{M}^{-1} \mathrm{~s}^{-1}\right)$. Values of the rate constants were estimated from Monte Carlo simulations using the kinetic model in Figure $6 a$ as described in detail previously for GluR1 and GluR4 channels (Robert and Howe, 2003). The parameters $k_{-1}$ and $k_{-1}{ }^{*}$ are the dissociation rate constants in the absence and presence of $100 \mu$ m cyclothiazide ( $k_{-1}$ was not determined for the T686 mutants with glutamate). When values are not given, they are the same as the value to the left in the same row. The experimental measurements that primarily constrained the values for the various rate constants are indicated by the superscripts. n.d., Not determined.

${ }^{a}$ Deactivation time constants.

${ }^{b}$ Desensitization time constants.

' $P_{\text {open }}$ and burst length measurements for GluR2-Q (Robert and Howe, unpublished observations).

${ }^{d}$ Kinetics of entry into desensitization at low glutamate (Robert and Howe, 2003).

${ }^{e}$ Concentration-response data for peak currents.

${ }^{f}$ Kinetics of recovery from desensitization.

${ }^{9}$ Relative amplitude of plateau and peak currents.

${ }^{h}$ Concentration-response data in cyclothiazide.

relative increases in the size of the plateau current (Table 1). In contrast, the mutations decreased the $m$ values obtained from the fits to the glutamate results. For T686A, the recovery time course is nearly exponential, and the relative increase in plateau current is substantially larger than the relative decrease in the time constant of recovery (Table 1).

Recently, we argued that the time course of recovery is determined by two sequential resensitization steps that correspond to the rate at which interactions that stabilize binding domain closure disengage and allow glutamate to dissociate (Robert and Howe, 2003). The faster recovery seen with mutations at T686 (which are predicted to destabilize binding domain closure) are generally consistent with this proposal and support the view that the stability of cleft closure is one determinant of both the rate at which AMPA receptors recover from desensitization and the rate at which they escape desensitization during sustained agonist applications. The nearly exponential recovery from glutamateevoked desensitization seen for T686A channels suggests that desensitization is sufficiently destabilized that at equilibrium most of the mutant channels are in the D1 states and spend little time in the D2 states (Bowie and Lange, 2002; Robert and Howe, 2003).

\section{The stability of binding cleft closure is a major determinant of affinity}

To characterize further the effects of the T686 mutations, we interpreted the various results in the context of a kinetic model that we showed previously accounted well for a variety of results obtained for wild-type GluR1 and GluR4 channels (Robert and Howe, 2003). This model is illustrated in Figure $6 a$ and contains five closed states, three open states, and eight desensitized states that differ in the number of subunits occupied by glutamate and the number of transitions they are removed from the similarly occupied closed state (because we were not interested in modeling the slow phase of recovery, the D0 state was disconnected in the simulations done here). Monte Carlo simulations were used to estimates values for the various rate constants for GluR2-wt and T686 mutant channels, as described previously (Robert and Howe, 2003).

The largest effect of the T686 mutations was to reduce the apparent affinity of glutamate binding. Although shifts in concentration-response curves can reflect alterations in either affinity or gating (Colquhoun, 1998), the results were not reproduced well in our simulations by changes in $\beta$ and $\alpha$, the rate constants 

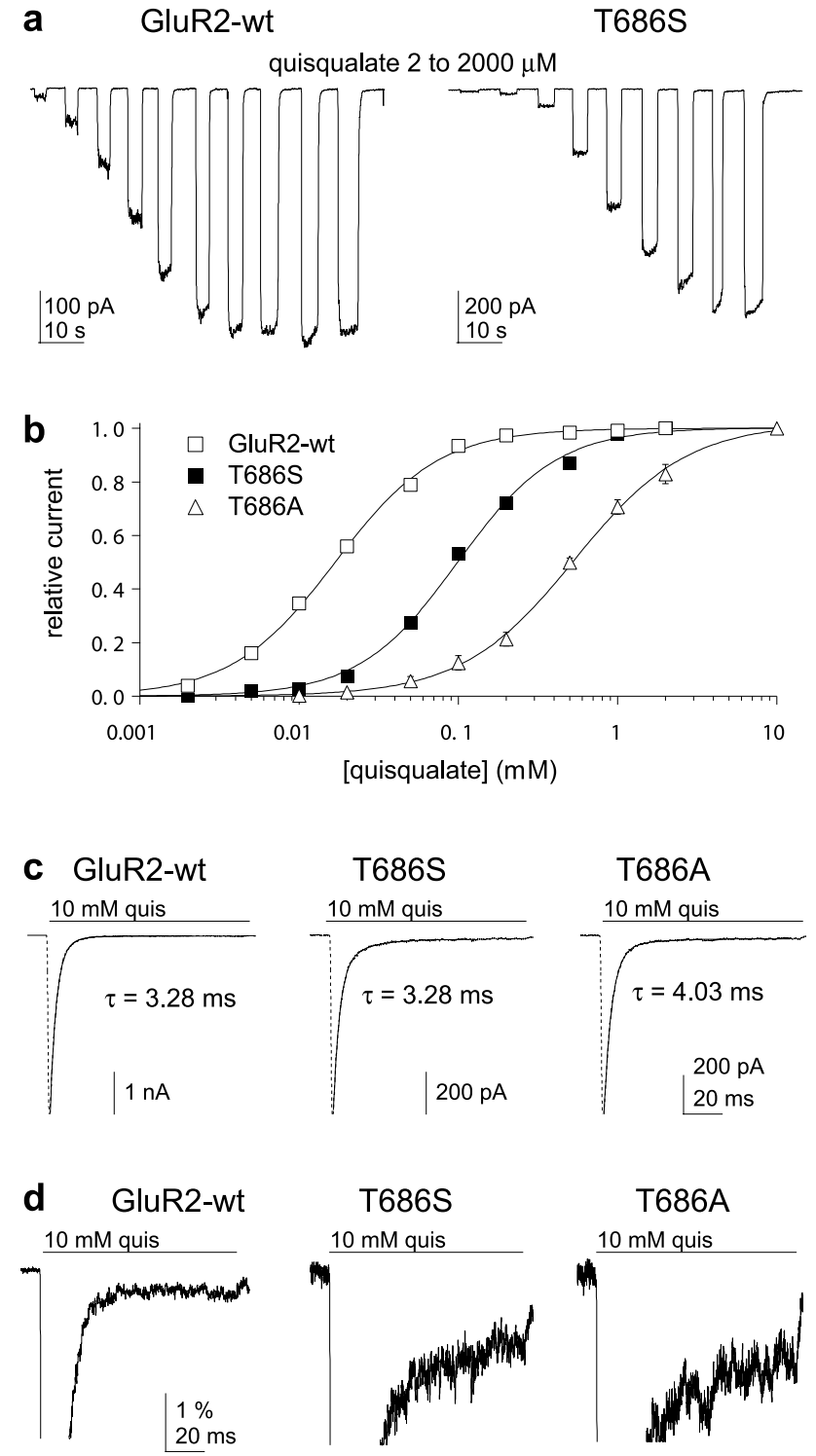

Figure 3. The effect of the mutations on quisqualate-evoked currents. $\boldsymbol{a}, \boldsymbol{b}$, Representative (a) and mean $(\boldsymbol{b})$ concentration-response data for quisqualate on GluR2-wt and the T686 mutants in the presence of $100 \mu \mathrm{m}$ cyclothiazide. The mean data ( $4-6$ patches per data point) were fitted with Hill-type equations (smooth curves). The $\mathrm{EC}_{50}$ and $n_{\mathrm{H}}$ values estimated from these fits are given in Table 1.c, Currents evoked by sustained applications of $10 \mathrm{~mm}$ quisqualate (quis) with desensitization intact. The biexponential fits (solid curves) to the decays of the currents gave the indicated time constants for the predominant fast component. $\boldsymbol{d}$, Examples of steady-state plateau currents recorded for GluR2-wt, T686S, and T686A channels after scaling the peak currents (off-scale) to have the same amplitude. The amplitude calibration is given as a percentage of the amplitude of the peak current.

for channel opening and closing. Because in our model (and most other published models) activation and desensitization occur in parallel (Vyklicky et al., 1991; Raman and Trussell, 1995; Robert et al., 2001), decreases in the $\beta / \alpha$ ratio sufficient to reproduce the observed rightward shifts in $\mathrm{EC}_{50}$ values would result in much faster desensitization, contrary to our observations that the rate of desensitization is unaltered or somewhat slowed. In total, the phenotype of the mutants suggests the shifts in $\mathrm{EC}_{50}$ values reflect a real increase in the rate at which glutamate dissociates, and simulations confirmed that the rightward shifts in $\mathrm{EC}_{50}$ values and faster deactivation seen with the T686 mutants were reproduced well by increases in $k_{-1}$ (Table 2 ).
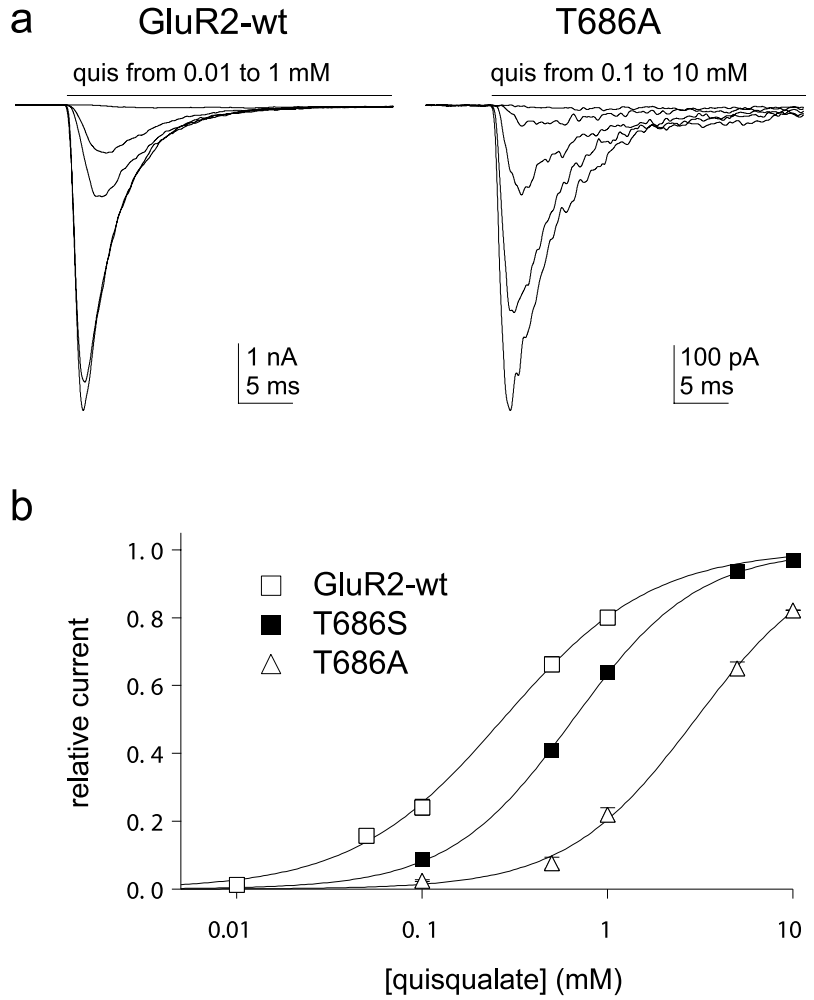

Figure 4. The T686 mutations increase $\mathrm{EC}_{50}$ values for peak currents. $\boldsymbol{a}$, Currents evoked in response to sustained applications of quisqualate (quis) in the absence of cyclothiazide in patches containing GluR2-wt (left) and T686A (right) channels. $\boldsymbol{b}$, Mean concentration-response data for peak quisqualate-evoked currents for GluR2-wt and the T686 mutants. Each point represents data from four to six patches. In each patch, the currents were normalized to the amplitude of the current evoked by $10 \mathrm{~mm}$ quisqualate. The $\mathrm{EC}_{50}$ and $n_{\mathrm{H}}$ values obtained from the Hill-type fits (smooth curves) to the results are given in Table 1.

Although neither T686 nor E402 appear to participate directly in coordination of bound glutamate, space-filling models of the S1S2 structure indicate that glutamate is entirely solvent inaccessible when the binding cleft is closed, and the crystallographic data strongly suggest that glutamate can only dissociate at an appreciable rate when the cleft opens. The rate of glutamate dissociation (Fig. $6 a, k_{-1}$ ) therefore reflects the combined rate of two sequential transitions. These transitions are illustrated in diagram form in Figure $6 b$ for agonist (yellow sphere) binding to a single S1S2 dimer (domains 1 and 2 of each monomer colored cyan and magenta, respectively). Here the rate constants $k_{+1}$ and $k_{-1}$ govern agonist binding to the open cleft conformation, whereas the rate constants $C C$ and $C O$ are the rate constants for binding cleft closure and opening, respectively. As illustrated, only receptors with closed binding domains have any appreciable probability of gating. The large effect of the T686 mutations on apparent affinity, although T686 does not make direct contact with agonists, suggests that the increases in $k_{-1}$ seen with the T686 mutants actually reflect increases in the value of $C O$ (attributable to destabilization of binding cleft closure).

Changes in the stability of binding cleft closure also provides an explanation for the well known effects of cyclothiazide to slow deactivation and decrease agonist $\mathrm{EC}_{50}$ values (Partin et al., 1996), neither of which is accounted for by simply slowing entry into desensitization. Cyclothiazide binds at, and stabilizes, the dimer interface (Sun et al., 2002). We propose that this stabilization also stabilizes binding cleft closure, which in turn directly slows the rate at which glutamate and other agonists dissociate. 


\section{The T686 mutations reveal differences in efficacy between glutamate and quisqualate}

In our formal kinetic model (Fig. 6a), changes in $k_{-1}$ will not alter channel open probability at saturating agonist concentrations, i.e., differences in $k_{-1}$ will not influence agonist efficacy. However, if the open/closed transition for the binding cleft is included (as in Fig. 6b), increases in the rate of binding cleft opening (CO) would be expected to reduce equilibrium efficacy if the ratio CC/CO is such that fully occupied channels spend an substantial fraction of their time in the open-cleft conformation. If the different potencies of quisqualate and glutamate in part reflect different values of $C O$ (quisqualate $<$ glutamate), then the T686 mutations should reduce the efficacy of glutamate more than that of quisqualate. We therefore compared the currents evoked in the same patches by concentrations of quisqualate $(10 \mathrm{~mm})$ and glutamate $(50 \mathrm{~mm})$ that were saturating (or nearly so) for GluR-wt and the T686 serine and alanine mutants. To simplify the comparison, steady-state currents were recorded after slowing desensitization with $100 \mu \mathrm{M}$ cyclothiazide.

As shown repeatedly for a variety of native and recombinant channels, quisqualate and glutamate produced similarly sized currents when applied to patches containing GluR2-wt channels (Fig. 6c). In contrast, glutamate consistently gave smaller population responses than quisqualate for both T686S and T686A channels (Fig. 6c). For GluR2-wt, the mean ratio of the current amplitudes evoked by $50 \mathrm{~mm}$ glutamate and $10 \mathrm{~mm}$ quisqualate was $0.98 \pm$ 0.03 ( $n=5$ patches). The corresponding ratios for T686S and T686A channels were $0.74 \pm 0.03$ and $0.44 \pm 0.02$, respectively ( $n=5$ and 6 patches). The results indicate that the T686 mutations reduce the efficacy of agonists, and they suggest that the efficacy of quisqualate and glutamate differ, although both appear to be full agonists for wild-type or native AMPA receptors.

To confirm that the T686 mutations alter agonist efficacy by destabilizing binding cleft closure, it would be useful to show that the results are predicted by a kinetic model in which the values that we estimate for $k_{-1}$ (Table 2) are instead taken as values for $C O$. The number of states required if the cleft-closure step is included makes a complete treatment too complicated to be feasible; however, the situation at high agonist concentration can be approximated by first calculating the probability that an occupied subunit adopts a conformation that allows current flow (see Materials and Methods, Predicting the effect of the mutations on agonist efficacy). In the absence of desensitization, this is equivalent to determining the steady-state probability that a subunit is in state 3 in Figure 6b. If it is further assumed that subunits gate independently, this probability can then be used in the binomial equation to calculate the mean current for a large population of channels (for d GluR2-wt

$10 \mathrm{mM}$ quis
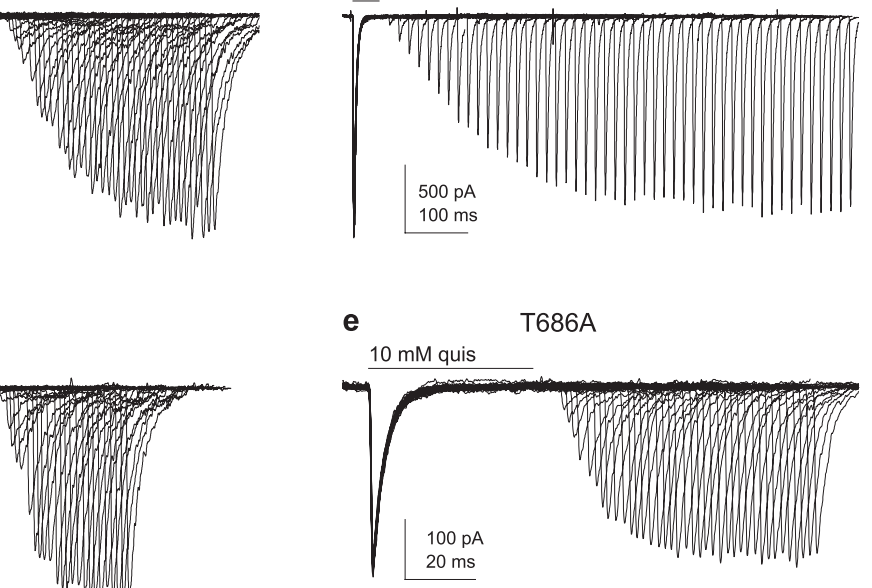

f

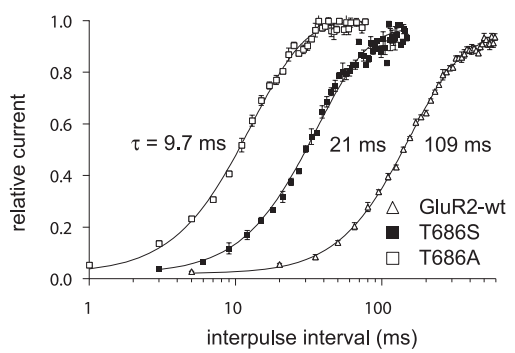

terpulse interval $(\mathrm{ms})$

Figure 5. Mutations at T686 speed recovery from desensitization. $\boldsymbol{a}, \boldsymbol{b}$, Currents evoked by 50 mm glutamate (glu) in outsideout patches from cells expressing GluR2-wt $(\boldsymbol{a})$ and the T686S mutant $(\boldsymbol{b})$. The results are from two-pulse protocols in which an second applications gives the time course of recovery from desensitization. c, Mean recovery data for glutamate from four to six

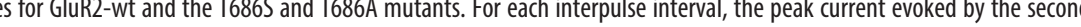
were fitted with Hodgkin-Huxley-type equations (smooth curves). $\boldsymbol{d}, \boldsymbol{e}$, Recovery results from two-pulse protocols with $10 \mathrm{~mm}$ is fivefold slower for the GluR2-wt results. $\boldsymbol{f}$, Mean recovery data for quisqualate from five to six patches for GluR2-wt and the T686S and T686A mutants. The pooled results were fitted with Hodgkin-Huxley-type equations (smooth curves). The time constants and $m$ values from the fits in $\mathbf{c}$ and $\boldsymbol{f}$ are given in Table 1.

details, see Materials and Methods). A similar approach was taken recently by Jin et al. (2003) to account for the different efficacies and single-channel behavior of a series of 5-substituted willardines that are partial agonists at AMPA receptors.

The results of the analysis are shown in Figure $6 d$ in which the ratio of the currents evoked by $50 \mathrm{~mm}$ glutamate and $10 \mathrm{~mm}$ quisqualate is plotted for three different values of $C C$ and a range of $\beta$ values. The values for $C O$ were set to the values for $k_{-1}$ given in Table 2 (in cyclothiazide), and $\alpha$ was set to $4000 \mathrm{~s}^{-1}$. The analysis predicts that the serine and alanine mutations should progressively decrease the efficacy of glutamate relative to quisqualate, and the predicted reductions are close to those observed for $C C=25,000$ to $50,000 \mathrm{~s}^{-1}$ and $\beta$ values of 5000 to $10,000 \mathrm{~s}^{-1}$. Although our estimates of $C C$ and $\beta$ are indirect, the values that we obtain are reasonable and give maximum open probabilities close to those observed in single-channel records for recombinant and native AMPA-type channels in the absence of desensitization (Rosenmund et al., 1998; Smith and Howe, 2000; Smith et al., 2000). 


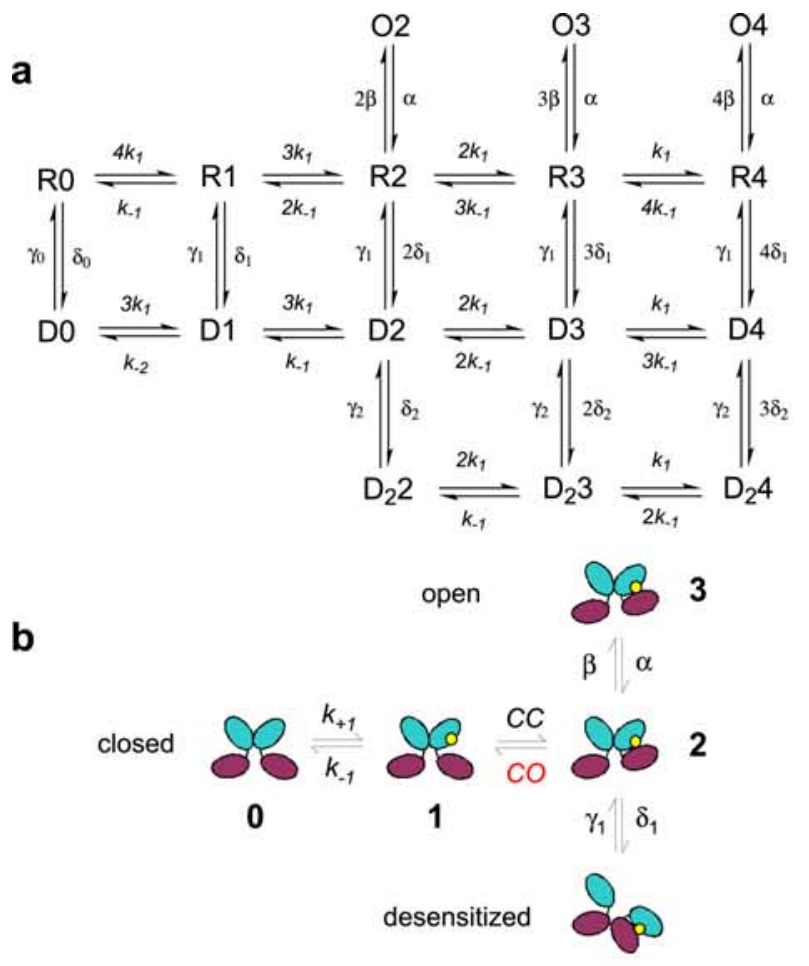

C
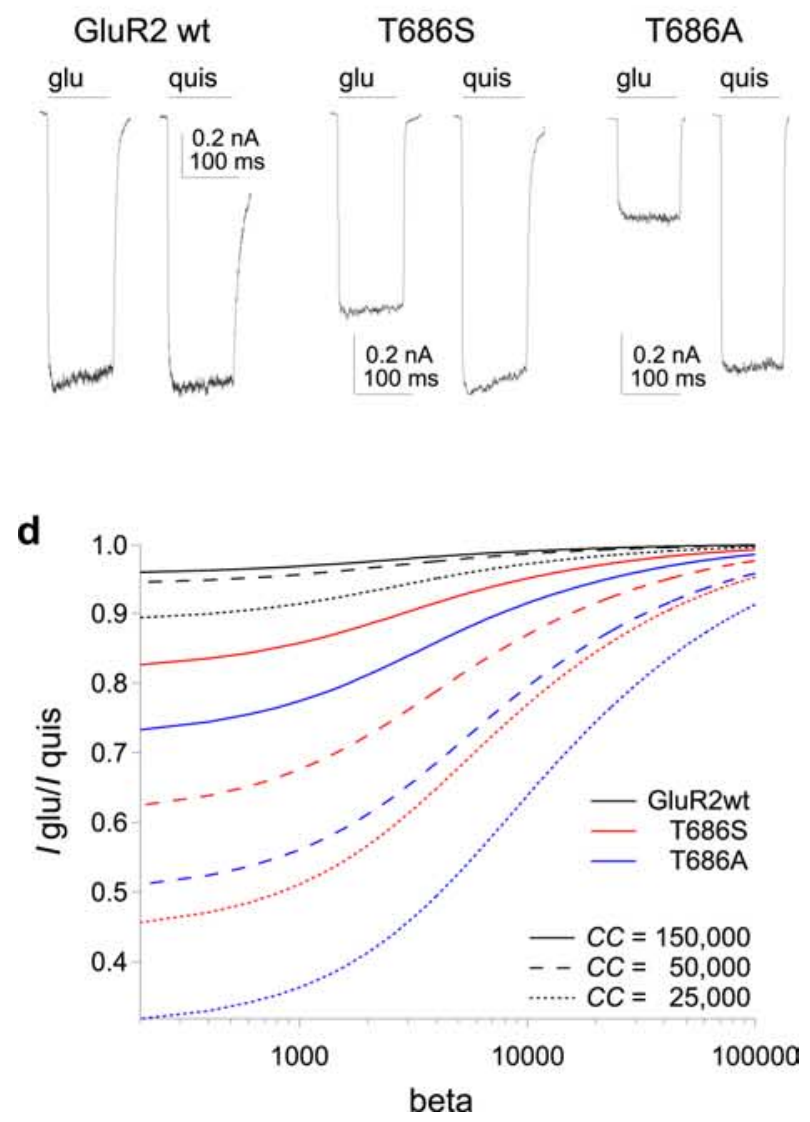

Figure 6. The T686 mutations increase the rate of glutamate dissociation by destabilizing binding cleft closure. $\boldsymbol{a}$, Kinetic model used to evaluate the effect of the T686 mutants on rate constants for transitions between distinct channel states. The model contains five closed states (R0 to R4), three open states ( 02 to 04 ), and eight desensitized states ( $D 0$ to $\left.D_{2} 4\right)$. The numbers in the first three rows of the model indicate the number of subunits occupied by glutamate. The
It is noteworthy that values of $C C$ and $\beta$ that reproduce the results for T686S predict that the T686A mutation should have a somewhat smaller effect than was actually observed. This deviation would be explained if, for glutamate, the T686A mutation reduces the value of $C O$ sufficiently so that dissociation from the open-cleft conformation contributes to the value of $k_{-1}$ that we obtain from our simulations. Assigning $C O$ this value of $k_{-1}$ would therefore underestimate its true value, and our analysis would underestimate the predicted reduction in glutamate efficacy. This would also explain why the shift in the $\mathrm{EC}_{50}$ value for T686A channels is greater for quisqualate than for glutamate.

The T686 mutations speed recovery by promoting reassembly of the dimer interface

The most intuitive explanation for the effect of the T686 mutations on recovery from desensitization is illustrated in Figure $7 a$. Here the T686 mutations destabilize binding cleft closure for both closed and desensitized channels and increase the values of both $C O$ and $\mathrm{CO}_{\mathrm{d}}$ (the rate of cleft opening for desensitized channels). In this case, the most likely sequence of events during recovery (red arrows) would be cleft opening of a desensitized channel, followed by the rapid dissociation of glutamate and reassembly of the dimer interface. In the context of our kinetic model, the two rate-determining steps during recovery would correspond to glutamate dissociation from states $\mathrm{D}_{2} 2$ and $\mathrm{D} 1$ (red arrows in kinetic scheme). This explanation does not account, however, for the direct correlation between the effect of the mutations on the rate of recovery and their effect to increase the relative size of the steady-state plateau current. In fact, because $\gamma_{1}$ must be substantially smaller than $C O_{\mathrm{d}}$ if cleft opening precedes resensitization (Fig. $7 a$ ), $C O_{\mathrm{d}}$ values that give the right recovery time course necessarily give plateau currents substantially smaller than those observed.

In contrast, the results are consistent with the route of recovery depicted in Figure $7 b$. Here cleft opening occurs after resensitization, and the T686 mutations speed recovery by increasing the rate of resensitization and reassembly of the dimer interface. Increases in $\gamma_{1}$ and $\gamma_{2}$, (Fig. $6 a$ ), the rate constants for the two resensitization steps, reproduced well both the faster recoveries seen with the mutants and the increased relative size of the plateau currents (Fig. 7c,d; Table 2).

\footnotetext{
$\leftarrow$ subscript 2 in the bottom row indicates that these desensitized states contain two subunits with closed binding domains ((Robert and Howe, 2003). $\boldsymbol{b}$, Diagram illustrating the sequence of events that results in AMPA receptor gating. The ligand binding domains of each subunit in a receptor dimer are shown (domains 1 and 2 of each subunit colored cyan and magenta, respectively), and agonist is depicted as a yellow sphere. At the left, both binding clefts are open, and the dimer interface is intact. Agonist binding to the open cleft conformation of one subunit is governed by the rate constants $k_{+1}$ and $k_{-1}$ and is followed by closure of the binding cleft. This closed-cleft/closed-channel conformation (state 2) puts strain on the dimer interface and is unstable. The instability is partially relieved by channel opening (state 3) or, more fully, by slippage of the dimer interface and desensitization (Sun et al., 2002). The T686 mutations increase the rate at which agonists dissociate by increasing the value of the rate constant $\mathrm{CO} . \mathrm{C}$, Steady-state currents evoked in the same patches by $50 \mathrm{~mm}$ glutamate (glu) and $10 \mathrm{~mm}$ quisqualate (quis) for GluR2-wt, T686S, and T686A channels. $\boldsymbol{d}$, Predicted efficacy of glutamate (relative to quisqualate) as a function of the rate constant for channel opening $(\beta)$. It was assumed that efficacy is related to the steady-state probability of channels being in state 3 in $\boldsymbol{b}$ (rather than states 0,1, or 2 ), that subunits gate independently, and that unitary conductance correlates with the number of subunits with closed binding domains (for details, see Materials and Methods). Calculations were performed for GluR2-wt, T686S, and T686A channels for three different values of the rate constant for cleft closure $(C()$, with the rate constant for cleft opening (CO) set to the values given in Table 2 for $k_{-1}$ in the presence of $100 \mu \mathrm{m}$ cyclothiazide. The glutamate and quisqualate concentrations were 50 and $10 \mathrm{~mm}$, respectively.
} 
a
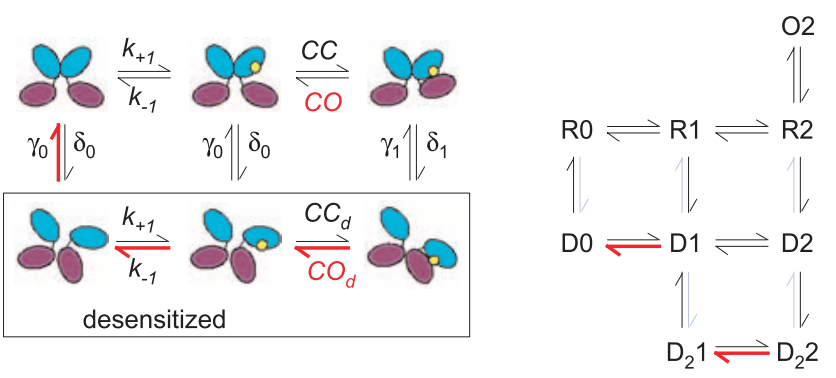

b
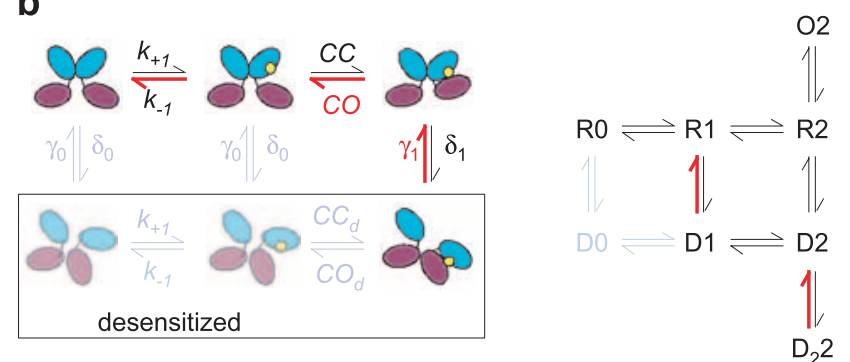

C

d
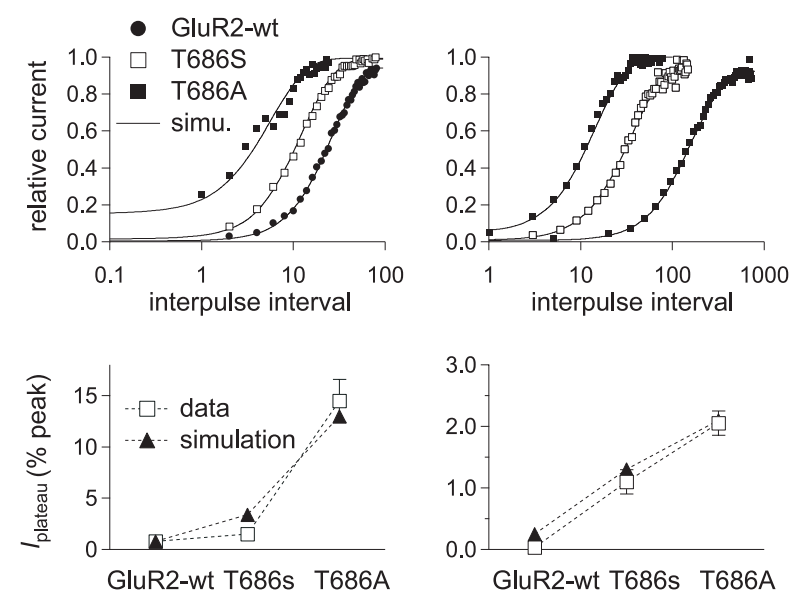

Figure 7. The T686 mutations speed recovery by increasing the rate of resensitization. $\boldsymbol{a}$, Diagram (left) and partial kinetic model (right) showing one possible sequence of transitions (red arrows) that occurs for a desensitized channel during recovery. Opening of the binding cleft determines the time course of recovery and precedes dissociation of glutamate and reassembly of the dimer interface. The T686 mutations speed recovery by increasing the value of the rate constant $\mathrm{CO}_{\mathrm{d}} \cdot \boldsymbol{b}$, The rate of resensitization determines the recovery time course, and the T686 mutations increase both this rate $\left(\gamma_{1}\right)$ and the rate at which closed channels open their binding domains ( $(0)$. In the partial kinetic models shown in $\boldsymbol{a}$ and $\boldsymbol{b}$, red arrows indicate the two rate-determining steps during recovery. In the diagrams and models, blue arrows are unlikely transitions, and blue states (and the pale diagram states) are unlikely to be visited. $c$, Simulated (simu.) recovery time courses (smooth curves) for GluR2-wt and the T686S and T686A mutants predicted for glutamate (left) and quiqualate (right) from the model in Figure $6 a$ and the rate constants in Table 2. The simulated curves were scaled to give the correct extent of recovery at the longest intervals tested experimentally. The curves are superimposed on the mean experimental data from Figure 5, c and $f$. $\boldsymbol{d}$, Experimental (open squares) and simulated (filled triangles) peak-to-plateau current ratios for GluR2-wt and the T686 serine and alanine mutants for glutamate (left) and quiqualate (right).

\section{Discussion}

\section{The T686 mutations destabilize binding cleft closure}

Although our results do not exclude other explanations, several observations support the conclusion that the primary effect of the T686 mutations is to decrease the stability of binding cleft closure. First, the available S1S2 structures indicate that T686 and E402 participate in a cross-cleft hydrogen bond, and neither T686 nor E402 directly contact glutamate or quisqualate (Armstrong and Gouaux, 2000; Jin et al., 2002). Substitution of serine for threonine is a conservative mutation that would be expected to weaken, but not completely disrupt, the T686/E402 interaction, whereas this interaction should be absent in the T686A mutant. Our observations that T686A produces similar, but larger, effects than T686S are consistent with these predictions. Second, the S1S2 structures indicate that glutamate and quisqualate interact similarly with sites in the binding pocket and that both agonists produce similar amounts of binding cleft closure (Armstrong and Gouaux, 2000; Jin et al., 2002). If the mutations altered the way ligands interact with the binding pocket, the effects on glutamate and quisqualate should be similar. Although the T686 mutations increase $\mathrm{EC}_{50}$ values for both agonists, the quantitative effects of the mutations on the two sets of concentration-response curves differ, and the mutations reveal differences in efficacy that have not been observed for wild-type or native channels. Third, the effects on affinity can be quantitatively accounted for by changes in the stability of cleft closure, if $k_{-1}$ in our simulations (using the model in Fig. $6 a$ ) is determined primarily by the value of the rate constant CO. Fourth, the effect of the mutations to reduce the efficacy of glutamate (relative to quisqualate) is predicted if the inherent stability of binding cleft closure contributes to the different potency of the two agonists.

\section{The stability of cleft closure influences affinity}

Previous work on the S1S2 structures suggested that the different apparent affinities of glutamate and quisqualate might reflect differences in the detailed interactions that their $\gamma$-substituents make with residues in subsite F of the binding pocket (Jin et al., 2002). In the glutamate-bound S1S2 structures, subsite F is occupied by a water molecule (W4). In the quisqualate-bound structures, the same position is occupied by an oxygen attached to the oxadiazolidine ring of quisqualate, and dissociation of the $\gamma$-substituent requires the breaking of an additional ligand-protein hydrogen bond. One other difference in the two S1S2 structures, however, involves the trans peptide bond between residues D651 and S652. It was noted previously that this bond was "flipped" in AMPA-bound S1S2 structures, a reorientation that allows the backbone carbonyl groups of these domain 2 residues to participate in two additional hydrogen bonds with residues in domain 1 (Armstrong and Gouaux, 2000). This peptide bond flip was only distinguished in one of the three protomers in the glutamate-bound crystal structure, whereas it is present in four of the five S1S2-quisqualate complexes (Armstrong and Gouaux, 2000; Jin et al., 2002). If, as suggested for AMPA (Armstrong and Gouaux, 2000), quisqualate is more effective than glutamate at promoting these cross-cleft interactions, this would be consistent with the idea that more stable binding cleft closure contributes to the greater potency of quisqualate.

The idea that the stability of cleft closure contributes to the rate at which agonists dissociate was discussed in previous papers on the GluR2 S1S2 structure, and the likely contribution of the interaction between T686 and E402 to closed-cleft stability was noted (Armstrong et al., 1998; Armstrong and Gouaux, 2000). Two phases in the kinetics of glutamate binding to the isolated ligand binding core were detected by Abele et al. (2000), who suggested that glutamate "docking" to the open-cleft conformation was fast and was followed by a slower "locking" step. The authors did not relate the "locked" conformation of the GluR4 isolated binding core to any functional channel state, and the kinetics of "unlocking" that they reported are much slower than the values we suggest here for the rate of cleft opening. Interest- 
ingly, however, the slow dissociation rate constant predicted $K_{\mathrm{D}}$ values for binding to the isolated binding core, and mutations at the residue equivalent to E402 in GluR4 increased the rate of unlocking by threefold to fivefold (Abele et al., 2000) and produced similar reductions in the affinity of glutamate binding (Lampinen et al., 1998).

Recently, the large increase in apparent affinity produced by the pore mutation lurcher was suggested to reflect the effect of the mutation to stabilize the closed-cleft conformation (Klein and Howe, 2004). Like lurcher, cyclothiazide and a leucine-totyrosine mutation at the dimer interface reduce desensitization, slow deactivation, and cause leftward shifts in agonist $\mathrm{EC}_{50}$ values (Partin et al., 1996; Stern-Bach et al., 1998; Robert et al., 2001; Sun et al., 2002). Consistent with previous work (Partin et al., 1996), our simulations show that cyclothiazide-associated shifts in $\mathrm{EC}_{50}$ values are best accounted for by changes in $k_{-1}$, changes we suggest reflect stabilization of the closed-cleft/closed-channel conformation. Alterations in the stability of binding cleft closure may be a common mechanism by which residues that are not directly involved in ligand coordination can influence agonist potency.

\section{The stability of cleft closure influences efficacy and entry into desensitization}

The different effects the T686 mutations have on the efficacy of glutamate and quisqualate provide additional evidence that the primary effect of the mutations is to destabilize cleft closure. The values we estimate for $C O$ predict the effect of the mutations on the relative amplitude of the currents evoked by saturating concentrations of glutamate and quisqualate for plausible values of $C C$ and $\beta$ (Fig. $6 d$ ).

A signature property of AMPA receptors is that they display concentration-dependent substate gating (Rosenmund et al., 1998; Smith and Howe, 2000). In a recent study on a series of 5 -substituted willardine partial agonists (which produce different amounts of domain closure in S1S2 structures), the relative efficacies measured for steady-state ensemble currents closely predicted the relative proportion of single-channel openings to small, intermediate, and large conductance levels (Jin et al., 2003). In our work, efficacy is related to the steady-state distribution of occupied subunits in the states numbered 1, 2, and 3 in Figure $6 b$, and efficacy is directly given by the probability of finding an individual subunit in state 3 at saturating agonist concentrations. For fully occupied T686 mutants, the efficacy values predict that the probability of all four binding domains being closed is lower for glutamate than quisqualate, and the smaller maximal responses predicted for glutamate result because it is the number of subunits with closed binding domains that correlates with unitary conductance. If this interpretation is correct, the T686 mutants should spend less time at the largest conductance level at saturating agonist concentrations, and smaller open levels should be more prevalent with glutamate than quisqualate. Thus, both incomplete and unstable cleft closure may have similar effects on gating at the single-channel level. However, the stability of cleft closure can influence both affinity and efficacy, whereas there is no relationship between the extent of cleft closure and affinity (Jin et al., 2003).

The slowing of desensitization seen with the T686 mutants, and the greater slowing seen with glutamate than quisqualate, are also consistent with destabilization of binding cleft closure. Because the channels can only desensitize at any appreciable rate from the closed-cleft state, the cleft is likely to open before the channels desensitize once the value of $C O$ exceeds the value of $\delta$
(Fig. 6b). The values our simulations give for $C O$ and $\delta$ (Table 2) predict that this effect should be greatest for the T686A mutant in the presence of glutamate, as was observed.

\section{The T686 mutations speed recovery from desensitization}

Both T686 mutations speed recovery from desensitization and increase the relative size of the plateau currents. These results indicate that the mutations destabilize desensitized states and promote reassembly of the dimer interface (Sun et al., 2002). We concluded previously that subunit-subunit (or interdomain) interactions occur during desensitization that trap glutamate in one subunit of each dimer (Robert and Howe, 2003). The present results are consistent with this view and indicate that reassembly of the dimer interface must occur before glutamate can dissociate from these subunits.

\section{Conclusions}

Considerable evidence suggests that closure of the AMPA receptor binding cleft is the initial conformational change that occurs after agonist binding, resulting in a transition state in which both the binding cleft and the channel are closed. Our results support the idea that it is primarily the rate at which the binding cleft opens that determines the rate at which agonists dissociate. We also show that, if cleft closure precedes subsequent gating steps, decreases in the stability of cleft closure can also decrease efficacy and slow desensitization. The 10 - and 20 -fold reductions in the stability of cleft closure produced by the serine and alanine mutants were accompanied by substantial reductions in glutamate efficacy. This result suggests that, during evolution, the stability of cleft closure has been tuned so that glutamate dissociates as rapidly as possible (as required for a fast transmitter) yet remains a full agonist at wild-type AMPA receptors.

\section{References}

Abele R, Keinanen K, Madden DR (2000) Agonist-induced isomerization in a glutamate receptor ligand-binding domain. A kinetic and mutagenetic analysis. J Biol Chem 275:21355-21363.

Armstrong N, Gouaux E (2000) Mechanisms for activation and antagonism of an AMPA-sensitive glutamate receptor: crystal structures of the GluR2 ligand binding core. Neuron 28:165-181.

Armstrong N, Sun Y, Chen GQ, Gouaux E (1998) Structure of a glutamatereceptor ligand-binding core in complex with kainate. Nature 395:913-917.

Armstrong N, Mayer M, Gouaux E (2003) Tuning activation of the AMPAsensitive GluR2 ion channel by genetic adjustment of agonist-induced conformational changes. Proc Natl Acad Sci USA 100:5736-5741.

Ayalon G, Stern-Bach Y (2001) Functional assembly of AMPA and kainate receptors is mediated by several discrete protein-protein interactions. Neuron 31:103-113.

Banke TG, Bowie D, Lee H, Huganir RL, Schousboe A, Traynelis SF (2000) Control of GluR1 AMPA receptor function by cAMP-dependent protein kinase. J Neurosci 20:89-102.

Bowie D, Lange GD (2002) Functional stoichiometry of glutamate receptor desensitization. J Neurosci 22:3392-3403.

Colquhoun D (1998) Binding, gating, affinity and efficacy: the interpretation of structure-activity relationships for agonists and of the effects of mutating receptors. Br J Pharmacol 125:924-947.

Dingledine R, Borges K, Bowie D, Traynelis SF (1999) The glutamate receptor ion channels. Pharmacol Rev 51:7-61.

Dzubay JA, Jahr CE (1999) The concentration of synaptically released glutamate outside of the climbing fiber-Purkinje cell synaptic cleft. J Neurosci 19:5265-5274.

Jin R, Horning M, Mayer ML, Gouaux E (2002) Mechanism of activation and selectivity in a ligand-gated ion channel: structural and functional studies of GluR2 and quisqualate. Biochemistry 41:15635-15643.

Jin R, Banke TG, Mayer ML, Traynelis SF, Gouaux E (2003) Structural basis 
for partial agonist action at ionotropic glutamate receptors. Nat Neurosci 6:803-810.

Jonas P, Major G, Sakmann B (1993) Quantal components of unitary EPSCs at the mossy fibre synapse on CA3 pyramidal cells of rat hippocampus. J Physiol (Lond) 472:615-663.

Klein RM, Howe JR (2004) Effects of the lurcher mutation on GluR1 desensitization and activation kinetics. J Neurosci 24:4941-4951.

Koike M, Tsukada S, Tsuzuki K, Kijima H, Ozawa S (2000) Regulation of kinetic properties of GluR2 AMPA receptor channels by alternative splicing. J Neurosci 20:2166-2174.

Lampinen M, Pentikainen O, Johnson MS, Keinanen K (1998) AMPA receptors and bacterial periplasmic amino acid-binding proteins share the ionic mechanism of ligand recognition. EMBO J 17:4704-4711.

Mano I, Lamed Y, Teichberg VI (1996) A venus flytrap mechanism for activation and desensitization of $\alpha$-amino-3-hydroxy-5-methyl-4isoxazole propionic acid receptors. J Biol Chem 271:15299-15302.

Mansour M, Nagarajan N, Nehring RB, Clements JD, Rosenmund C (2001) Heteromeric AMPA receptors assemble with a preferred subunit stoichiometry and spatial arrangement. Neuron 32:841-853.

Partin KM, Fleck MW, Mayer ML (1996) AMPA receptor flip/flop mutants affecting deactivation, desensitization, and modulation by cyclothiazide, aniracetam, and thiocyanate. J Neurosci 16:6634-6647.

Patneau DK, Mayer ML (1991) Kinetic analysis of interactions between kainate and AMPA: evidence for activation of a single receptor in mouse hippocampal neurons. Neuron 6:785-798.

Raman IM, Trussell LO (1995) The mechanism of alpha-amino-3-hydroxy5-methyl-4-isoxazolepropionate receptor desensitization after removal of glutamate. Biophys J 68:137-146.

Robert A, Howe JR (2003) How AMPA receptor desensitization depends on receptor occupancy. J Neurosci 23:847-858.
Robert A, Irizarry SN, Hughes TE, Howe JR (2001) Subunit interactions and AMPA receptor desensitization. J Neurosci 21:5574-5586.

Rosenmund C, Stern-Bach Y, Stevens CF (1998) The tetrameric structure of a glutamate receptor channel. Science 280:1596-1599.

Sekiguchi M, Nishikawa K, Aoki S, Wada K (2002) A desensitizationselective potentiator of AMPA-type glutamate receptors. Br J Pharmacol 136:1033-1041

Smith TC, Howe JR (2000) Concentration-dependent substate behavior of native AMPA receptors. Nat Neurosci 3:992-997.

Smith TC, Wang LY, Howe JR (2000) Heterogeneous conductance levels of native AMPA receptors. J Neurosci 20:2073-2085.

Stern-Bach Y, Russo S, Neuman M, Rosenmund C (1998) A point mutation in the glutamate binding site blocks desensitization of AMPA receptors. Neuron 21:907-918.

Sun Y, Olson R, Horning M, Armstrong N, Mayer M, Gouaux E (2002) Mechanism of glutamate receptor desensitization. Nature 417:245-253.

Swanson GT, Kamboj SK, Cull-Candy SG (1997a) Single-channel properties of recombinant AMPA receptors depend on RNA editing, splice variation, and subunit composition. J Neurosci 17:58-69.

Swanson GT, Gereau RW, Green T, Heinemann SF (1997b) Identification of amino acid residues that control functional behavior in GluR5 and GluR6 kainate receptors. Neuron 19:913-926.

Swanson GT, Green T, Heinemann SF (1998) Kainate receptors exhibit differential sensitivities to (S)-5-iodowillardiine. Mol Pharmacol 53:942-949.

Uchino S, Sakimura K, Nagahari K, Mishina M (1992) Mutations in a putative agonist binding region of the AMPA-selective glutamate receptor channel. FEBS Lett 308:253-257.

Vyklicky Jr L, Patneau DK, Mayer ML (1991) Modulation of excitatory synaptic transmission by drugs that reduce desensitization at AMPA/kainate receptors. Neuron 7:971-984. 